Cahiers Société

\title{
L'imaginaire et le symbolique : Castoriadis et le structuralisme de Lévi-Strauss
}

\section{Stéphane Vibert}

Numéro 1, mai 2019

Castoriadis et les sciences sociales

URI : https://id.erudit.org/iderudit/1068420ar

DOI : https://doi.org/10.7202/1068420ar

Aller au sommaire du numéro

\section{Éditeur(s)}

Collectif Société

ISSN

2562-5373 (imprimé)

2562-5381 (numérique)

Découvrir la revue

Citer cet article

Vibert, S. (2019). L'imaginaire et le symbolique : Castoriadis et le structuralisme de Lévi-Strauss. Cahiers Société, (1), 35-61. https://doi.org/10.7202/1068420ar
Résumé de l'article

Élaborant une critique interne du marxisme qui le conduit à proposer une pensée de la création radicale et de l'imaginaire instituant, Castoriadis va dès les années 1960 amorcer la critique cinglante d'une mouvance intellectuelle alors en vogue, le structuralisme, considéré comme une exemplification " panlogique " du déterminisme scientiste qu'il honnit. La critique castoriadienne du structuralisme conjoindra dès lors une contestation de ses soubassements épistémologiques à une condamnation de ses implications politiques. Cette contribution se propose d'approfondir la confrontation théorique entre Castoriadis et Lévi-Strauss, l'auteur central de la méthode structuraliste, en identifiant trois lieux spécifiques aptes à mettre en lumière les désaccords ontologiques irréductibles qui semblent ressortir de leurs oeuvres respectives : tout d'abord, la nature et la finalité de l'anthropologie philosophique et sociale postulée à titre de fondement épistémologique ; ensuite, la question nodale du « sens ", articulée à l'appréhension des concepts de " code " et de " signification "; enfin, la conception du social-historique comme totalité ou comme culture qui distingue fortement les deux analyses. 


\section{L'imaginaire et le symbolique : Castoriadis et le structuralisme de Lévi-Strauss}

Stéphane VIBERT

Université d'Ottawa

Le dialogue intellectuel entre Castoriadis et Lévi-Strauss ne semble jamais réellement avoir eu lieu. Les deux œuvres, gigantesques, relèvent pourtant d'une ambition intellectuelle similaire, au moins en creux : toutes deux ont en quelque sorte quitté les rivages du marxisme afin de proposer une conception englobante alternative de l'humanité sociale et de son histoire, ainsi que des fondements imaginaires ou symboliques de son accès au sens. Il faut noter que ces deux pensées, qui ont partagé une même scène publique dans la France de la seconde moitié du $\mathrm{XX}^{\mathrm{e}}$ siècle, ont également eu en commun de ne jamais s'arrimer à un champ disciplinaire clos et unique : touchant la philosophie, l'ethnologie, l'histoire ou la sociologie, voire la linguistique et l'art, elles développent toutes deux, de façon très dissemblable, une véritable anthropologie générale, au sens fort du terme. En raison du contexte intellectuel français des années 1950-1960, rattaché au prestige universitaire du structuralisme et à son hégémonie théorique, il ne serait sans doute pas totalement erroné d'affirmer que les critiques résolues et récurrentes de Castoriadis à son encontre ont pu un temps participer à sa marginalisation et à sa relative méconnaissance. Jusqu'à la fin des années 1980, Castoriadis réitérera ses désaccords avec l'approche structuraliste en général, d'ailleurs davantage avec la vulgate d'une nébuleuse structuraliste formée à partir de l'association discutable de « maîtres »-Lacan, Barthes, Foucault, Althusser - qu'avec la théorie lévi-straussienne elle-même, sur laquelle il ne paraît pas revenir après la mise au point initiale élaborée et proposée dans L'institution imaginaire de la société. Ainsi, dans sa lecture des événements de mai 1968, le structuralisme est-il présenté comme une «idéologie pseudo-scientifique ${ }^{1}$ » sans aucune influence sur les brèches créatrices ouvertes par le mouvement révolutionnaire. Dans un texte conséquent sur la psychanalyse, Castoriadis vilipendait également ce qu'il nommait «l'Idéologie Française ${ }^{2}$ » (avec deux majuscules), incarnée dans un structuralisme voué au paradoxe épistémologique, puisque fondé sur un projet scientiste et rationa-

\footnotetext{
${ }^{1}$ Cornelius Castoriadis, «Les mouvements des années soixante », dans Les carrefours du labyrinthe, $I V$. La montée de l'insignifiance, Paris, Seuil, 1996, p. 31.

${ }^{2}$ Cornelius Castoriadis, «La psychanalyse, projet et élucidation », dans Les carrefours du labyrinthe, I, Paris, Seuil, 1978, p. 153. Voir également « Les mouvements des années soixante », op. cit., p. 31.
} 
liste indigent et suranné - la quête d'une combinatoire universelle -, tout en se payant le luxe d'une dénonciation de la Raison occidentale étriquée. La subordination de la signification au code algébrique aurait ainsi notamment eu comme conséquences premières de " persuader le sujet de son inexistence ou de son irresponsabilité, le passiviser devant les "structures"; fermer la question de la pensée et de la vérité ; nier l'histoire [...] donc aussi exclure d'avance la question de la possibilité d'une autre société3 ». C'est d'ailleurs la déliquescence subséquente de ce paradigme structuraliste 4 pris comme un tout, comme un "effet de mode» dépassant de loin l'œuvre de Lévi-Strauss lui-même, qui selon Castoriadis favorisera un retour du refoulé politique sous des formes pathologiques, que ce soit la libération du désir, la dénonciation du langage comme fasciste ou l'extrapolation des réseaux de pouvoir à l'ensemble des relations sociales.

La critique castoriadienne du structuralisme conjoindra donc constamment une contestation de ses soubassements épistémologiques à une condamnation de ses implications politiques. On sait d'ailleurs que ces deux versants ne s'avèrent jamais assurément dissociés chez Castoriadis, puisque, si le fameux mot d'Honneth voyant dans son travail un "sauvetage ontologique de la révolution » semble excessif, voire fallacieux, il n'en reste pas moins que l'insistance sur l'imprévisible création historique adossée à l'imaginaire instituant entend bien laisser totalement ouverts les champs du possible et de la transformation radicale, à distance d'une philosophie héritée - marxisme compris - engoncée dans les rets du déterminisme ensemblisteidentitaire. Et c'est bien au titre privilégié d'incarnation momentanément prééminente de ce paradigme scientiste que le structuralisme lévi-straussien perçu comme panlogicisme constituera une cible explicite de critique.

Il convient d'emblée de préciser que, dans les limites inhérentes à ce texte, plusieurs points majeurs du problème ne pourront pas être abordés, par exemple l'histoire et la nature du mouvement structuraliste lui-même, en son évolution propre et son hétérogénéité foncière, ou encore le rapport fondamental à la théorie psychanalytique lacanienne, qui se trouve en quelque sorte au croisement de la réflexion castoriadienne et de l'extension de la perspective lévi-straussienne. C'est pourquoi, dans la perspective féconde ouverte par une problématique qui s'attelle à faire travailler l'œuvre de Castoriadis à l'aune de diverses confrontations théoriques, il sera plus raisonnable et heuristique de partir des analyses originelles assez sommaires mais éclairantes proposées dans L'institution imaginaire de la société, élaborées au courant des années 1960-1970, en précisant la divergence épistémologique qui s’y exprime avec le structuralisme, de façon parfois complexe, notamment dans la discus-

\footnotetext{
3 Cornelius Castoriadis, «La psychanalyse, projet et élucidation », dans Les carrefours du labyrinthe, I, op. cit., p. 154.

4 «[... le "structuralisme" s'est dissous, personne n'ose plus l'invoquer et les plus habiles, comme Foucault, prétendent qu'ils n'en sont plus et/ou qu'ils n'en ont jamais été » : Cornelius Castoriadis, «Les mouvements des années soixante », dans Les carrefours du labyrinthe, IV, op. cit., p. 33.
} 
L'imaginaire et le symbolique | Stéphane VIBERT

sion qui concerne le rapport de complémentarité hiérarchique entre les dimensions imaginaires et symboliques du monde social-historique. Cet exposé introductif nous permettra dans un second temps d'approfondir la confrontation théorique entre Castoriadis et Lévi-Strauss, en centrant notre attention sur trois problèmes significatifs aptes à mettre en lumière les désaccords ontologiques irréductibles qui semblent ressortir de leurs œuvres respectives : le premier problème concerne la nature et la finalité de l'anthropologie philosophique et sociale postulée à titre de fondement épistémologique; le deuxième lieu de divergence touche la question du «sens", articulée à celle du code et de la norme; enfin, nous aborderons en un troisième temps la conception générale du social-historique qui se dégage des deux analyses, à partir de la notion holiste de « totalité sociale » comme point d'achoppement majeur.

\section{Castoriadis et la critique fondamentale du structuralisme : l'imaginaire et le symbolique}

On sait que L'institution imaginaire de la société constitue en réalité, ainsi que le rappelle Castoriadis dans sa préface, un collage entre deux textes de facture et de temporalité très différentes. La première partie, intitulée «Marxisme et théorie révolutionnaire ", a été élaborée progressivement dès la fin des années 1950, avant d'être publiée dans la revue Socialisme ou Barbarie d'avril 1964 à juin 1965. Quant à la seconde, qui touche plus directement "l'imaginaire social et l'institution", elle concrétise une réflexion plus tardive, au tournant des années 1960-1970, jusque certainement la remise du manuscrit, en 1974. C'est dire qu'il faut prendre en compte, durant ces années de concrétisation et de formalisation des concepts centraux de Castoriadis, la dimension évolutive de sa pensée, dans un contexte lui-même mouvant, qui voit justement une certaine déshérence du paradigme structuraliste, dans la période qui suit les soubresauts de Mai 68 notamment. Ce hiatus entre les deux parties induit un rapport différent au structuralisme lévi-straussien : s'il est discuté directement et explicitement dans la première partie, notamment à travers une analyse des distinctions et complémentarités entre les dimensions symboliques et imaginaires de l'être social-historique, il n'est plus abordé qu'à titre marginal et secondaire dans la seconde partie, le «symbolisme" disparaissant même quasi entièrement de l'exposé au profit des significations imaginaires sociales.

Si la critique radicale à l'encontre du marxisme - qui aboutit à un constat péremptoire et définitif : " le marxisme est mort comme théories $»-s$ 'adosse à une mise en exergue des soubassements problématiques qui le constituent comme tel (rationalisme, objectivisme, déterminisme, économicisme), il faut alors pour Castoriadis construire le versant positif de cette critique, ce qui, au détour d'une discussion sur l'aliénation comme phénomène social et la relance du projet d'autonomie, le conduit aux prémices d'une conception originale du social-historique. C'est dans ce dévelop-

5 Cornelius Castoriadis, L'institution imaginaire de la société, Paris, Seuil, 1999 [1975], p. 93. 
pement, qui dissipe l'illusion de la transparence à soi et insiste sur l'impossibilité absolue pour tout individu d'éliminer l'inconscient comme pour toute société de se priver d'institution, qu'une claire référence au vocabulaire structuraliste apparaît, à travers la reprise du concept central de « symbolique» :

De même que l'individu ne peut saisir ou se donner quoi que ce soit - pas plus le monde que soi-même - en dehors du symbolique, une société ne peut se donner quoi que ce soit en dehors de ce symbolique au second degré que représentent les institutions ${ }^{6}$.

Mais la reprise explicite du concept n'empêche pas une critique fondamentale de certains des aspects épistémologiques les plus importants de la méthode structurale, de ses postulats et de ses effets. Il faut noter que dans son argumentation, Castoriadis prend d'abord comme cible l'approche fonctionnaliste et son entreprise réductionniste, interprétant toute culture comme une réponse à des besoins vitaux universels, et ce afin de déployer, comme réponse à ce matérialisme biologisant, une véritable théorie du "symbolisme» - terme utilisé sans contraste apparent avec celui de « symbolique » substantivé. La thèse est forte et limpide : « Tout ce qui se présente à nous, dans le monde social-historique, est indissociablement lié au symbolique7 ». Et de citer en exemple des actes individuels ou collectifs comme «le travail, la consommation, la guerre, l'amour, l'enfantement» qui seraient impossibles s'ils n'étaient tissés dans un réseau symbolique. En rappelant que les institutions ne peuvent exister que dans le symbolique, que ce soit l'économie, le droit, le politique ou la religion, qui apparaissent socialement comme "des systèmes symboliques sanctionnés » attachant des symboles (ordre du signifiant) à des signifiés (des représentations et des significations), Castoriadis se retrouve ainsi au plus proche de la célèbre définition de la culture donnée par Lévi-Strauss dans son introduction au recueil de Marcel Mauss, Sociologie et anthropologie, publié en 1950 : « un ensemble de systèmes symboliques au premier rang desquels se placent le langage, les règles matrimoniales, les rapports économiques, l'art, la science, la religion ${ }^{»}$. Dans la suite immédiate de l'exposé, Castoriadis s'accorde avec diverses autres idées fortes de Lévi-Strauss sur le sujet, parmi lesquelles la mise en évidence de six caractéristiques du symbolique, très succinctement évoquées, à savoir : la consistance, la socialisation, la totalisation, l'étayage, la continuité et la surabondance. Consistance : le symbolisme s'avère relativement indépendant de la fonctionnalité. Socialisation: l'individu rencontre toujours devant lui un monde symbolique suréminent et déjà-là. Totalisation : chaque société constitue son propre ordre symbolique. Étayage : cette élaboration symbolique par une société donnée n'est jamais absolue, puisqu'elle doit prendre sa matière dans la nature particulière qui l'environne. Continuité : un systè-

\footnotetext{
6 Ibid., p. 169.

7 Ibid., p. 174.

8 Claude Lévi-Strauss, «Introduction à Marcel Mauss », dans Marcel Mauss, Sociologie et anthropologie, Paris, Presses Universitaires de France, p. xix.
} 
me symbolique doit s'édifier sur les ruines des édifices symboliques précédents, sans possibilité de rupture totale. Enfin, surabondance : "par ses connexions naturelles et historiques virtuellement illimitées, le signifiant dépasse toujours l'attachement rigide à un signifié précis et peut conduire à des lieux totalement inattendus ${ }^{~} \gg$. Il s'agit là, comme on le sait, d'une des plus complexes et notoires propositions de LéviStrauss, selon laquelle « le signifiant précède et détermine le signifié10 », ce qui occasionne d'ailleurs le «surplus de signification ${ }^{11}$ » qui débouche sur l'existence des fameux « signifiants flottants » de type mana.

Si le symbolisme, notamment attaché au langage et aux institutions, apparaît comme primordial dans cette compréhension de la société, c'est qu'il permet, par son autonomie et sa cohérence propre, de se distancier de toutes les théories diverses et multiples d'un réalisme " naïf » et positiviste qui réduisent le social à sa fonctionnalité ou sa rationalité. Tout en s'accrochant à l'historique et au naturel, et tout en participant au rationnel, le symbolique les dépasse tel un ordre de sens autonome, qui défie toutes les dichotomies habituelles : ni librement choisi ni imposé de l'extérieur; ni médium neutre et transparent ni milieu opaque et impénétrable. C'est justement dans ces interstices, en fondant un nouvel usage lucide ou réfléchi des symboles qui structurent une société, que peut réellement s'incarner la liberté tant collective qu'individuelle. Dans certaines formulations originelles, Castoriadis fait montre d'un optimisme révolutionnaire certain, clairement volontariste et artificialiste quant à ce rapport, affirmant par exemple qu'une " nouvelle société créera de toute évidence un nouveau symbolisme institutionnel », laquelle réduirait ainsi l'autonomisation aliénante du symbolisme pour le ramener à "l'adéquation au contenu ${ }^{12}$ », cette «maitrise du symbolisme des institutions » ne posant en dernière instance " pas des problèmes essentiellement différents de ceux de la maîtrise du langage ${ }^{13} » \ldots$

Ceci étant dit, l'introduction par Castoriadis de la dimension de l'imaginaire engage ici immédiatement une relativisation foncière de la fonction symbolique, et modifie du tout au tout l'ordonnancement épistémologique ainsi proposé. Il existe entre imaginaire et symbolique un rapport de complémentarité hiérarchique: l'imaginaire doit certes utiliser le symbolique pour exister et pour s'exprimer, mais tout symbolisme présuppose d'emblée la capacité imaginaire « de voir dans une chose ce qu'elle n'est pas, de la voir autre qu'elle n'est $14 »$. À ce stade, le concept d'imaginaire dernier ou radical inclut à la fois l'imaginaire effectif, qui désigne les produits de la faculté imaginante, et le symbolique, qui en structure le contenu par des liens d'identification, de participation ou de causation entre signifiants et signifiés, entre symboles et choses. On le devine, c'est dans ce dépassement de la logique

9 Cornelius Castoriadis, L'institution imaginaire de la société, op. cit., p. 181.

10 Claude Lévi-Strauss, «Introduction à Marcel Mauss », op. cit., p. xxxii.

${ }^{11}$ Ibid., p. xlix.

${ }^{12}$ Cornelius Castoriadis, L'institution imaginaire de la société, op. cit., p. 189.

13 Ibid., p. 190.

14 Ibid., p. 191. 
classificatoire en direction de significations à la fois socialement englobantes, culturellement particulières et ontologiquement immotivées que se trouve la rupture théorique fondamentale entre Castoriadis et les hypothèses structuralistes, ces vues « qui ne veulent voir dans l'institution que le symbolique (et identifient celui-ci au rationnel15) ». Or, sur ce point, la thèse de Castoriadis est sans équivoque :

On ne peut pas [...] comprendre les institutions simplement comme un réseau symbolique. Les institutions forment un réseau symbolique mais ce réseau, par définition, renvoie à autre chose que le symbolisme. Toute interprétation purement symbolique des institutions ouvre immédiatement ces questions : pourquoi ce système-ci de symboles, et pas un autre; quelles sont les significations véhiculées par les symboles, le système des signifiés auquel renvoie le système de signifiants; pourquoi et comment les réseaux symboliques parviennent-ils à s'autonomiser ${ }^{16}$.

Fondamentalement, Castoriadis décèle une " aporie philosophique » à la source de l'hypothèse ontologique proposée par Lévi-Strauss quant à la réduction possible $d u$ sens, qui résulterait en dernière instance d'un non-sens, c'est-à-dire d'une combinaison d'éléments en eux-mêmes non signifiants, selon le fonctionnement inconscient et universel d'un esprit, lui-même identifié au cerveau matériel. Cette analyse aboutirait à une conséquence tautologique : «l'activité de l'esprit consiste à se symboliser soimême en tant que chose dotée de pouvoir symbolisateur ${ }^{17} »$.

L'insistance sur cette activité universelle de l'esprit manque en réalité la compréhension du social-historique, qui exige de transcender l'analyse structuraliste du symbolisme au moins sur trois plans :

i. Le « choix » qu'une société fait de son symbolisme, c'est-à-dire les raisons qui poussent à investir tels ou tels phénomènes afin de penser le monde en se pensant soi-même.

ii. La saisie des « significations 》 véhiculées par les oppositions structurales, qui seraient ontologiquement premières par rapport à leur mise en forme selon une logique combinatoire : " considérer le sens comme simple "résultat" de la différence des signes, c'est transformer les conditions nécessaires de la lecture de l'histoire en conditions suffisantes de son existence ${ }^{18} »$. Autrement dit, la combinaison des signes résulte autant de la visée de sens que l'inverse, et l'histoire humaine révèle d'évidence une véritable création du symbolisme, une genèse du sens par la production de nouveaux systèmes de signifiés et signi-

\footnotetext{
15 Ibid., p. 197.

16 Ibid., p. 205-206.

17 Ibid., p. 205, n. 33.

18 Ibid., p. 208.
} 
fiants, mais aussi un usage relativement indéterminé de tout système symbolique déjà existant, tant par sur-détermination possible des symboles (plusieurs signifiés pour un même signifiant) que sursymbolisation du sens (même signifié porté par plusieurs signifiants). Ici, l'option structuraliste conduit donc à " éliminer le sens ", en le réduisant à une saveur subjective, liée à une intériorité monadique psychologique et affective.

iii. La critique de l'autonomisation aliénante du système symbolique institutionnel, qui ne s'avère possible que parce tout discours humain, par-delà sa structuration symbolique, s'institue comme visée de sens, un sens perçu, pensé ou imaginé. Or, l'approfondissement par Castoriadis de cette question du sens l'amène à le comprendre, de façon complexe, comme un «noyau indépendant de tout discours et de toute symbolisation 19 ", un "schème organisateur-organisé » qui est « condition opérante de toute représentation ultérieure », sans pouvoir être saisi autrement qu'a posteriori, à partir de ses manifestations, que celles-ci se déclinent sous la forme de perceptions, valorisations ou investissements d'affects. Ces «articulations dernières » qu'une société impose au monde, faites de «schèmes organisateurs qui sont conditions de représentatibilité de tout ce que cette société peut se donner ${ }^{20}$ » ne peuvent donc être saisies que " de manière dérivée et oblique ${ }^{21} »$, comme « le ciment invisible tenant cet immense bric-à-brac de réel, de rationnel et de symbolique qui constitue toute société et comme le principe qui choisit et informe les bouts et les morceaux qui y seront admis ${ }^{22} »$. Il s'agit donc de ce que Castoriadis nommera bientôt «significations imaginaires sociales centrales", qui délimitent une collectivité à partir d'un certain «ordre du mon$\mathrm{de}^{23} \gg$ qu'elle pose comme étant sien.

Il est aisé de comprendre qu'en ce lieu précis, circonscrit dès les années 1960, réside l'opposition fondamentale et irréconciliable entre les perspectives de Castoriadis et Lévi-Strauss. Dans la seconde partie de L'institution imaginaire de la société, écrite ultérieurement, Castoriadis ne revient essentiellement sur ce qu'il nomme abruptement « l'assassinat structuraliste ${ }^{24}$ » - la réduction de la création imaginaire sociale magmatique à une structure logique de type ensidique - qu'en deux occasions. D'abord dans le cadre de sa cartographie des deux voies principales de l'ontologie héritée, caractérisée justement par sa négation de la spécificité imaginaire du social-

\footnotetext{
19 Ibid., p. 211.

20 Ibid., p. 215.

${ }^{21}$ Ibid., p. 216.

22 Idem.

23 Ibid., p. 224.

24 Ibid., p. 336.
} 
historique. À distance du type physicaliste, qui réduit société et histoire à la nature biologique de l'homme et prend la forme des divers fonctionnalismes, le structuralisme se retrouve dans le type "logiciste ", étonnamment aux côtés des philosophies de l'histoire qui en étaient pourtant le principal adversaire épistémologique et politique dans les années 1960. La division apparaît d'ailleurs quelque peu problématique dans le cas du structuralisme, si on se rappelle que le « logicisme » qui lui est imputé repose en dernière instance dans la capacité physiologique du cerveau humain à coder selon des classifications opératoires, et correspond donc à une faculté de nature biologique. Selon Castoriadis néanmoins, si les téléologies progressistes, par le déploiement de la raison, la matière ou la nature, incarnent le finalisme logiciste et rationaliste qui sied à un développement historique prédéterminé, le structuralisme quant à lui effectue le geste inverse, et par la suppression de l'histoire qu'il suppose, se voit jugé par Castoriadis comme «la forme la plus pauvre de logicisme25». Le structuralisme rendrait compte de la totalité de l'histoire humaine par la répétition d'une même opération logique, les sociétés n'apparaissant alors que comme des combinaisons différentes d'un nombre fini d'éléments discrets. Le jugement est péremptoire : le structuralisme ferait preuve d'une "naïveté désarmante » en ignorant tant la raison d'existence que les transformations historiques affectant les oppositions binaires élémentaires, comme masculin/féminin, nord/sud, haut/bas, sec/humide, etc., censées aller de soi alors même qu'elles relèvent de significations imaginaires valorisées par telle ou telle société.

Le second et dernier retour critique sur le structuralisme aura une profondeur théorique beaucoup plus imposante. Il se précise à l'occasion de l'exposé castoriadien des déterminations du legein et du teukhein par lesquelles se déploie la logique ensembliste-identitaire au sein des institutions d'une société. La discussion est évidemment importante, puisque la démonstration de Castoriadis consiste d'abord à reconnaître que l'étayage sur la première strate naturelle des institutions humaines implique effectivement, à l'instar du postulat structuraliste, qu'il existe bien une « organisation fixe et stable d'une partie du monde homologue à l'organisation de l'homme en tant que simple vivant ${ }^{26}{ }^{\prime}$. Cependant, à la différence de Lévi-Strauss pour qui le codage effectué par l'esprit sur la nature s'avère une opération de traduction horizontale, sans changement de niveau ontologique, Castoriadis affirme que l'étayage effectué par la société modifie la nature même du réel prélevé : «Ce qui y appartient est repris dans et par le magma de significations que la société institue, il est, par là, transsubstantié ou ontologiquement altéré. Il est altéré dans son mode d'être - en tant qu'il est et n'est que du fait de son investissement par la signification $27 »$. En d'autres mots, il n'est possible de connaître et présupposer l'organisation ensembliste qu'à partir de la signification, du moins quasiment, puisque

\footnotetext{
25 Ibid., p. 256.

26 Ibid., p. 345.

27 Ibid., p. 347-348.
} 
«l'organisation ensembliste naturelle comme telle n'est presque rien ${ }^{28}$ », une bonne partie du problème résidant néanmoins dans la nature et l'importance de ce "presque rien ». La visée de Castoriadis, quelles que soient les difficultés de sa position, consiste à récuser toute réduction causaliste rationnelle du monde social-historique, selon lui opérée par la démarche structuraliste et sa quête d'un tableau combinatoire universel, qui reconduirait l'idée ethnocentrique selon laquelle

la pensée mythique serait essentiellement pensée classificatoire, donc réductible aux rudiments de la logique ensembliste (les significations imaginaires comme saveurs, feux follets ou illusions partagées par les bons sauvages et les mauvais ethnologues29).

À cette occasion, Castoriadis se permet de paraphraser ironiquement en la retournant la critique portée par Lévi-Strauss contre le fonctionnalisme: "dire que les sauvages classifient, c'est un truisme (sans cela, ils ne parleraient pas); mais dire que pour l'essentiel ils ne font que classifier, c'est une absurdité30 ».

Par son analyse du legein comme dimension ensidique du représenter-dire social, à travers les opérations de pouvoir distinguer-choisir-poser-rassembler-compterdire ${ }^{11}$, Castoriadis retrouve inévitablement la structuration du langage comme code, décelant le soubassement ontologique de la perspective structuraliste. En effet, le langage « établit toujours des termes (éléments ensemblistes) et des relations pratiquement univoques (ensemblistes ou ensemblisables) entre termes ; il comprend et il institue toujours une relation d'univocité, ou identitaire ${ }^{2} »$. Reprenant les analyses de la linguistique structurale de Troubetskoï ou Jakobson, Castoriadis illustre cette dimension d'être-code tant par l'aspect matériel-abstrait du langage (système phonologique, classes grammaticales, types syntactiques, lexique) que par l'aspect significatif, dans les opérations de désignation ou nomination à prétention univoque, selon des propriétés séparées, fixes et stables. D'où une tension, soulignée par Castoriadis, entre ce code qui tend fictivement à se refermer sur lui-même, et la vocation des significations à renvoyer constamment à autre chose, dans un lexique indéfiniment ouvert. C'est alors autour de la « relation signitive 33 », relation signe-objet incompréhensible dans sa contingence sans la prise en compte d'un imaginaire social spécifique, que se présente la tension avec la réduction structurale. Car c'est non pas seulement «l'arbitraire du signe» qui s'avère fondamental, mais surtout l'arbitraire du phantasma social-historique, du sensible sans matière fondant la représentation collective sur des images et figures concrètes, productrices de réel. La "désignation »

\footnotetext{
28 Ibid., p. 349.

29 Ibid., p. 349-350

30 Ibid., p. 350.

31 Ibid., p. 351.

32 Ibid., p. 352.

33 Ibid., p. 362.
} 
L'imaginaire et le symbolique | Stéphane VIBERT

comme « institution originaire 34 » nécessite comme relation signitive un passage par des schèmes opérateurs, comme la séparation, la réunion ou la valorisation, sans jamais trouver d'explication véritable hors d'une création social-historique. En ce sens, la signification excède nécessairement le langage comme code, et l'imaginaire dépasse le symbolique, en entraînant la déterminité dans une altération indéfinie, productrice de formes autres. C'est bien en cette adjonction d'une dimension ontologique irréductible à la scientificité que s'affirme le contraste entre les propositions de Castoriadis et Lévi-Strauss.

\section{Esquisses d'une confrontation théorique en trois points}

Partir des nombreux éléments critiques, primordiaux ou secondaires, soulignés par Castoriadis à l'encontre des propositions lévi-straussiennes soulève évidemment diverses questions au plan méthodologique. Il faudrait discuter le fait, d'une part, que la lecture castoriadienne s'avère valide en sa compréhension générale et son interprétation particulière de l'œuvre, et d'autre part, qu'elle ne tord pas involontairement le sens de l'entreprise en négligeant certains aspects fondamentaux de la démarche de Lévi-Strauss, elle-même éminemment complexe et sujette à de nombreux débats depuis déjà plus d'un demi-siècle. Néanmoins, parmi les très nombreux points de controverse, il est possible de délimiter trois enjeux spécifiques, successivement de nature ontologique, épistémologique et sociopolitique : la nature et la finalité de la «philosophie sociale» des deux penseurs, pour la nommer ainsi ; la question du " sens ", articulée à celle du code et de la norme ; et enfin, la conception du socialhistorique qui se dégage des deux analyses.

\section{Quelle philosophie sociale et quelle science de l'homme?}

La profonde divergence qui saute aux yeux lorsqu'on examine la teneur des deux projets ressortit sans nul doute à leur visée philosophique respective. Tandis que Castoriadis répète inlassablement vouloir saisir, par-delà la tradition héritée, la « création et genèse ontologique dans et par le faire et le représenter/dire des hommes 35 ", c'est-à-dire le nouveau, l'inédit, l'imprévisible, Lévi-Strauss s'attache avant tout par la méthode structurale à découvrir des rapports d'invariance, à travers une connaissance du dispositif de différences et de relations réciproques entre éléments divers. Le passage par la structure indique la permanence d'un système aussi stable, régulier et intégrateur que possible, procédant par oppositions, inversions ou homologies, définissant une interdépendance des éléments identifiés, l'existence d'un " groupe de transformations », une possibilité prédictive quant à l'évolution du modèle, ainsi

34 Ibid., p. 366.

35 Ibid., p. 8. 
qu'une capacité à rendre compte de tous les faits observés ${ }^{36}$. Autrement dit, ainsi que l'indique Lévi-Strauss lui-même dans son célèbre débat avec Paul Ricœur en novembre 1963, il serait possible de comprendre son entreprise "comme une sorte d'inventaire des contraintes mentales, une tentative pour réduire l'arbitraire à un ordre, pour découvrir une nécessité, immanente à l'illusion de la liberté37», acceptant de la nommer positivement, sur la suggestion de Ricœur, « kantisme sans sujet transcendantal $38 »$. Si Castoriadis récuse également tout sujet transcendantal, qui réduirait nécessairement les diverses expressions historiques à des variations d'une logique intangible sous-jacente, il ne saurait en aucun cas être question d'une détermination a priori des conditions de possibilité d'une création humaine par une autoconstitution imaginative, laquelle précède et oriente la connaissance théorique qu'on peut en avoir.

D'une certaine façon, c'est autour de la définition même de la "science » que le désaccord se concrétise. La démarche structuraliste épouse sans ambiguité une méthode rationaliste et positiviste, que Lévi-Strauss a lui-même rapprochée d'une forme de matérialisme étendu. Elle tomberait donc à l'évidence dans la définition donnée par Castoriadis de la science galiléenne, afin d'en contredire le postulat ensemblisteidentitaire :

le programme d'un savoir constituant son objet comme processus en soi indépendant du sujet, repérable sur un référentiel spatiotemporel valant pour tous et privé de mystère, assignable à des catégories indiscutables et univoques (identité, substance, causalité), exprimable, enfin, dans un langage mathématique à la puissance illimitée, dont ni la préadaptation miraculeuse à l'objet ni la cohérence interne ne semblaient poser de question 39.

D'ailleurs, commentant le projet structuraliste visant à unifier par interrelation des structures différentes (linguistiques, économiques, politiques), Castoriadis va jusqu'à écrire que " parler d'homologie structurale ou d'appartenance commune à une structure englobante des oppositions phonématiques et des formes de pouvoir d'une société fait penser à un canular plutôt qu'à un programme de recherche 40 ». En effet, cet ambitieux dessein englobant laisserait en plan des problèmes aussi complexes et incontournables que l'hétérogénéité des niveaux de l'être du social, l'interrogation sur la raison des différences de structure ainsi que leur évolution et transformation dans le temps.

36 Selon le résumé des quatre conditions de l'analyse structurale précisées dans Marcel Hénaff, Claude Lévi-Strauss et l'anthropologie structurale, Paris, Belfond, 1991, p. 26-27.

37 Claude Lévi-Strauss, "Réponses à quelques questions », entretien du "groupe philosophique » d'Esprit avec Claude Lévi-Strauss, dans Esprit, vol. 11, n 322, nov. 1963, p. 630.

${ }^{8}$ Ibid., p. 633.

39 Cornelius Castoriadis, «Science moderne et interrogation philosophique », dans Les carrefours $d u$ labyrinthe, I, op. cit., p. 196.

40 Ibid., p. 264-265. 
Alors que Castoriadis fait appel à la notion d'étayage sur la première strate naturelle pour penser le fait que le réel soit représentable et organisable selon une logique ensidique tout en tenant compte de l'irréductibilité de la signification à la causation, la position de Lévi-Strauss paraît beaucoup plus radicale, en supprimant tout simplement l'écart ontologique. Il s'agit en quelque sorte d'un monisme naturaliste, dont la concrétisation au niveau de l'humain s'effectue par le biais d'un matérialisme cérébral. Ainsi que l'écrit Descola, Lévi-Strauss

n'a jamais varié $[\ldots]$ dans l'idée que la nature conditionne les opérations intellectuelles grâce auxquelles la culture reçoit un contenu empirique ni hésité à anticiper sur le moment où celle-ci pourrait être interprétée en termes purement organiques, comme le résultat naturel et le mode social d'appréhension des modifications de structure et de fonctionnement du cerveau ${ }^{41}$.

Toute vie sociale s'avère donc d'emblée marquée par deux déterminismes simultanés et complémentaires :

l'un de type techno-économique impose à la pensée des contraintes résultant du rapport qu'une société entretient avec un milieu particulier; l'autre reflète les exigences inhérentes au fonctionnement de l'esprit et se manifeste partout à l'identique, indépendamment des différences entre les milieux ${ }^{42}$.

Ces « deux natures » n'ont de fait pas à être réconciliées, puisqu'elles procèdent d'un isomorphisme initial entre langage et monde : «les propriétés structurales de la nature ne se distinguent pas dans leur essence des codes au moyen desquels le système nerveux les déchiffre, ni des catégories dont l'entendement se sert pour rendre compte des propriétés du réel43». Dès Les structures élémentaires de la parenté, Lévi-Strauss affirmait que la prohibition de l'inceste ne faisait que codifier des déterminations imposées par la nature selon des schèmes cognitifs préexistant aux normes qui les traduisent. Ces schèmes cognitifs - exigence de la règle, notion de réciprocité, caractère synthétique du don - ne sont jamais abordés directement par Castoriadis en tant que tels, puisqu'il les renvoie le plus souvent à des «trivialités », des universels formels qui ne disent rien de la spécificité humaine. Tout au plus reconnaît-il la valeur contraignante du mode abstrait d'opération ensemblisteidentitaire - tel le legein et le teukhein - qui sous-tend la réalité social-historique, obligeant à constater

l'existence d'un invariant historique que nous ne savons pas « expliquer»: l'histoire du langage et des langages affecte et

\footnotetext{
${ }^{11}$ Philippe Descola, «Les deux natures de Lévi-Strauss », dans Les Cahiers de l'Herne. Claude LéviStrauss, dir. Michel Izard, nº 82, Paris, Champs Flammarion, 2014, p. 264.

42 Ibid., p. 264.

43 Ibid., p. 268.
} 
altère les langages concrets, y compris leur base «matérielleabstraite » chaque fois spécifique, mais non pas le type général de leur organisation 44 .

Ceci étant dit, deux questions peuvent être soulevées pour ce premier thème, qui paraît de prime abord opposer abruptement les deux penseurs. Si Castoriadis tend à déconsidérer la prise en compte des universaux sociohistoriques, il n'en reste pas moins qu'il consacre une part importante de L'institution imaginaire de la société à détailler le fonctionnement du legein et du teukhein comme modalités ensidiques universelles et nécessaires de l'institution social-historique, tout en insistant sur leur relance dynamique par des significations imaginaires particulières. Ne serait-on donc pas en présence d'une tentative de déterminer des schèmes idéels et pratiques de la condition humaine, reliés à une certaine conception de la nature ? De même, lorsque Castoriadis évoque ce problématique étayage sur la première strate naturelle, toujours repris et modifié dans les significations imaginaires d'une société particulière, ne faut-il pas considérer que la «correspondance 45 » entre réel et pensée, une correspondance que Castoriadis comprend comme « un fait, un pur fait, un fait brut ${ }^{46}$ ", retrouve un "être-ainsi du monde» purement objectif et matériel, qui précède et détermine toute imagination, fût-elle la plus radicale : «le pour soi ne peut être qu'en créant $[\ldots]$ un monde propre suffisamment "analogue" à des traits du monde "extérieur" ; et une telle création est rendue possible par cela qu'aussi bien le monde propre du pour soi que le monde tout court comportent une dimension ensidique $47 \gg$. Affirmer comme ontologie du social-historique le surgissement de déterminations nouvelles n'élimine pas la question transcendantale, autrement dit les conditions de possibilité qui rendent cette émergence possible, même s'il s'agit de saisir alors quelle relation entretiennent entre elles les différentes formes créées au sein d'un Être en devenir.

Pour conclure provisoirement sur ce point, il convient de s'interroger sur la nature de ce mode ontologique qu'est le symbolisme, et sur son destin historique. Nous avons rapidement évoqué le fait que Castoriadis, tout en subordonnant le niveau symbolique (assimilé à l'ensidique) à l'institution imaginaire des significations, considère à la fois comme légitime et possible une maîtrise croissante de ces niveaux, grâce à une activité collective et individuelle toujours plus réflexive. Le projet d'autonomie, qui conjugue politique, philosophie et démocratie dans une visée constante d'élucidation, de délibération et de décision, infère donc au plan socialhistorique ce que la psychanalyse rend possible au plan individuel. Mais Castoriadis ne cessera de le rappeler: nulle transparence à soi ne s'avère possible, puisque la

44 Cornelius Castoriadis, L'institution imaginaire de la société, op. cit., p. 398.

45 Cornelius Castoriadis, «Fait et à faire », dans Les carrefours du labyrinthe, V. Fait et à faire, Paris, Seuil, 1997, p. 15.

46 Idem.

47 Ibid., p. 17. 
quête d'autonomie consiste justement en une praxis continuée et infinie, par conscientisation, réflexion et critique. Ceci étant dit, l'espoir de voir advenir une société autonome au sens fort du terme (révolutionnaire), incline Castoriadis dans certaines formulations à accentuer la possibilité d'un « usage lucide et réfléchi » étendu et explicite, gouverné par le logon didonai (rendre compte et raison) apte à « tout mettre en question 48 », jusqu'au système symbolique lui-même, langage compris. Cette " conquête progressive du symbolisme 49 ", de nature sans doute asymptotique, ne saurait évidemment l'évider, mais suppose une atténuation de son caractère aliénant, ainsi qu'un rapport autre aux significations imaginaires qui sous-tendent le travail social-historique. Or, ce problème se pose tout autrement chez Lévi-Strauss. Si la pensée symbolique, définie comme armature logico-sociale des représentations et comportements, déjà attestée dans la "pensée sauvage», constitue bien le mode inhérent à l'esprit humain qui rend compte de son adéquation à « la nature des choses $5^{0}$ ", la science moderne, notamment la biologie et les études cognitives, qui en est le déploiement contemporain, semble pouvoir rendre compte plus adéquatement de la structure du connaissable. En effet, là où la pensée symbolique confère immédiatement un sens global au monde, quitte à passer par des signifiants flottants vagues et indéterminés, la science entreprend un ouvrage méthodique de "péréquation du signifiant par rapport au signifié51 », ce qui laisserait penser, à l'instar de Marcel Hénaff, qu'à terme "la pensée symbolique pourrait disparaître tout à fait dans un univers où les savoirs objectifs auraient répondu à la totalité des signifiants disponibles 52 ». C'est pourquoi une théorie globale de la communication vient en dernière instance chez Lévi-Strauss se substituer à ce que Camille Tarot appelle le paradigme du «tout symbolique53», selon une compréhension extensive du concept, associé à tous les faits de culture (dits "symboliques »), en mettant sur un même plan des phénomènes aussi différents que le système linguistique, la parenté, les rapports économiques, les formes artistiques, les énoncés scientifiques ou les expressions religieuses. Plus largement encore que ce symbolique identifié à l'esprit humain classificateur, le paradigme communicationnel permet d'intégrer les codes non socioculturels découverts par la science, comme l'A.D.N. au fondement de l'ordre biologique lui-même, mais aussi le niveau cellulaire ou les systèmes de communication animale, révélant par là le fonctionnement homogène du réel aux différents niveaux de ses modes d'existence. On aperçoit là une thèse ontologique à la fois gigantesque et problématique, concevant l'ensemble de la réalité à travers la codification interne qui s'y révèle progressivement, pour un esprit humain qui en est

48 Cornelius Castoriadis, L'institution imaginaire de la société, op. cit. p. 189.

49 Idem.

50 Selon Lévi-Strauss, «L'esprit est aussi une chose, le fonctionnement de cette chose nous instruit sur la nature des choses : même la réflexion pure se résume en une intériorisation du cosmos. Sous une forme symbolique, elle illustre la structure de l'en-dehors ", cité dans Marcel Hénaff, Claude LéviStrauss et l'anthropologie structurale, op. cit., p. 186.

${ }^{1}$ Claude Lévi-Strauss, cité in ibid., p. 187.

$5^{2}$ Marcel Hénaff, Claude Lévi-Strauss et l'anthropologie structurale, op. cit., p. 187.

53 Camille Tarot, Le symbolique et le sacré. Théories de la religion, Paris, La Découverte, 2008, p. 349. 
L'imaginaire et le symbolique | Stéphane VIBERT

qui s'y révèle progressivement, pour un esprit humain qui en est lui-même la manifestation réflexive.

\section{La question du sens, entre imaginaire et symbolique}

On l'a déjà signalé, un terreau intellectuel et sociohistorique commun rapproche Lévi-Strauss et Castoriadis : c'est pour tous deux le double refus, ferme et argumenté, tant d'une philosophie de l'histoire, assimilée grossièrement à la téléologie hégéliano-marxiste, que du fonctionnalisme élémentaire fondé sur une théorie des besoins. Et pareillement pour Lévi-Strauss et Castoriadis, à des degrés bien dissemblables certes, c'est le recours à la psychanalyse et aux médiations culturelles qui va servir de levier initial à la critique du sujet pleinement conscient, transparent à luimême, siège de volonté et de rationalité, qu'il soit individuel comme dans le libéralisme ou collectif comme dans le marxisme orthodoxe. Il est étonnant d'ailleurs de se rappeler à quel point les intuitions originelles de Lévi-Strauss s'ancrent, par un cheminement différent, dans la même aversion que celle de Castoriadis, à savoir une récusation définitive de toutes les théories fonctionnalistes hégémoniques en socioanthropologie au mitan du $\mathrm{XX}^{\mathrm{e}}$ siècle. Au cœur de l'anthropologie classique, que ce soit chez Morgan, Malinowski, Murdock ou Steward par exemple, il existe une tendance forte à expliquer les formes d'organisation sociale et d'expression culturelle comme des réponses adaptatives à certaines contraintes biologiques ou des impératifs de subsistance. Pour Malinowski, toute culture doit satisfaire le système biologique des besoins, et toute réalisation culturelle impliquant l'utilisation d'artefacts et du symbolisme consiste dès lors en un rehaussement instrumental de l'anatomie humaine. De telles explications ramènent les primitifs à une vie au plus près des nécessités physiologiques, bien loin des complexités de la civilisation qui élabore des niveaux non immédiatement utilitaires.

Sans qu'on puisse faire autre chose qu'esquisser ce rapprochement, le détour par la phénoménologie, et notamment la philosophie de Merleau-Ponty, semble jouer un rôle non négligeable, émettant l'hypothèse d'une vie à quelque degré déjà esprit, à travers ses expressions sédimentées et objectivées que sont les institutions, les pratiques, les œuvres - autrement dit, un sens déjà-là, avant même d'être repris, abstrait, théorisé. Ainsi, reconnaissent tous deux Lévi-Strauss et Castoriadis, il existe toujours un sens généralisé, une rationalité contingente, un esprit objectif qui structure et oriente les formes culturelles et historiques, que le sujet se réapproprie partiellement sans jamais le surplomber et le saisir entièrement. C'est évidemment dans l'appréhension de ce "sens déjà-là » que diffèrent largement les propositions de Castoriadis et Lévi-Strauss, à partir d'une controverse qui sourd de la question du sens, dans son rapport au code et à la norme. La notion d'imaginaire permet à Castoriadis de critiquer le réductionnisme au symbolique de la démarche structuraliste, conçu dans son esprit comme logique ensidique de codage du réel par oppositions binaires, sur le modèle de la linguistique structurale. Construisant sa pensée 
sous l'égide d'un paradigme informationnel qui se nourrit des développements de l'informatique et de la cybernétique, Lévi-Strauss fait partie d'une entreprise collective visant à proposer une théorie générale de la communication à tonalité cognitiviste, dont les années 1940-1960 incarnent le moment inaugural. Si Castoriadis est entré en débat avec certains représentants de ce courant, comme Varela, à partir d'une réflexion sur l'autopoïèse, il a toujours contesté ce qu'il estimait être à l'occasion un appauvrissement du sens par réduction à une forme renouvelée de scientisme logiciste54. Le refus partagé par Lévi-Strauss et Castoriadis d'une philosophie rationaliste de la conscience ouvre sur deux visions opposées, voire contradictoires, du rapport entre sens et vérité. Là où pour l'approche structurale il y a sens parce que des ensembles intelligibles relevant de savoirs objectifs peuvent être entrevus à partir de l'analyse des données culturelles et historiques, Castoriadis place le sens en excès de cette histoire de la vérité, par sa reprise dans des significations imaginaires sociales, vecteurs d'affects, d'intention et de normativité. Le rapport de convenance ou de correspondance entre réalité physique et monde humain se voit donc toujours débordé par l'imaginaire, alors qu'a contrario, on trouve chez Lévi-Strauss un postulat d'homologie parfaite entre le fonctionnement spontané de l'esprit se saisissant des données empiriques livrées par les sens, d'une part, et, d'autre part, le matériau déjà structuralement codé de l'en-dehors, à l'instar des atomes, des molécules, des cellules ou des organismes eux-mêmes.

Ce rapport divergent à la question du sens résulte d'un désaccord sur le rapport entre symbolisme et imaginaire, et plus largement, d'un désaccord sur l'ontologie du social-historique. D'une part, nous y reviendrons, parce que la notion d'imaginaire s'avère inexistante chez Lévi-Strauss en tant que telle, sinon à titre implicite. D'autre part - mais la question est ici extrêmement complexe -, pour une raison qui n'est pas relevée par Castoriadis dans sa lecture critique (peut-être parce qu'elle ne peut se révéler que par un regard global porté sur l'œuvre, qui se poursuivra bien au-delà des analyses castoriadiennes). C'est que la référence au symbolisme chez Lévi-Strauss recèle des ambiguïtés foncières, n'étant ni explicitement défini, ni univoque dans ses emplois. Quelques commentateurs avisés, comme Marcel Hénaff, l’ont amplement souligné. D'un certain point de vue, la pensée de Lévi-Strauss ne renonce pas à une certaine forme, traditionnelle en socioanthropologie, d'analyse du symbolisme, tel qu'il s'incarne dans des institutions spécifiques, donnant sens et forme à une culture donnée, et ce à travers des médiations selon des domaines d'activité sociale significativement orientées : la famille, la politique, l'économie, la religion, etc. Mais d'autre part, les recherches de Lévi-Strauss, sous notamment l'influence de la linguistique, vont le conduire vers une définition fondamentale de la condition humaine en termes tout à fait distincts. Selon cette seconde acception, le propre d'un dispositif symboli-

\footnotetext{
54 Ceci n'empêche pas qu'il existe dans la réflexion castoriadienne une réelle influence de la pensée d'inspiration cybernétique, notamment dans la conceptualisation du vivant comme pour soi, tant pour l'autoréférentialité, l'autofinalité et la clôture systémique qui le caractérisent.
} 
que ne consiste pas à signifier, évoquer ou illustrer, mais d'abord et avant tout à opérer, selon un régime de performance quasi technique, à l'instar d'un rituel, d'une démonstration mathématique ou d'une variante musicale. Il y aurait donc chez LéviStrauss une tension dans la définition symbolique entre deux registres, l'un davantage mentaliste et l'autre plutôt pragmatique, les deux se rejoignant néanmoins dans une récusation identique de la représentation consciente, réflexive et conceptuelle. D'une part, en effet, la société humaine «n'advient que dans ce décrochement global que constitue l'émergence de l'ordre symbolique en tant que système de termes différentiels, condition de toute reconnaissance réciproque et de tout discours articulé, de toute possibilité de saisir un ordre du monde $55 »$. C'est en cela qu'on peut parler d'une « origine symbolique de la société ». Mais par ailleurs, à partir du moment où la société est posée comme culture, Lévi-Strauss paraît comprendre toutes les activités et productions comme symboliques, donnant ainsi une extension apparemment excessive au terme. Selon Hénaff, c'est par le concept d' « efficacité symbolique 56 », moteur d'un dispositif opératoire mobilisant des affects, que se réalise la jonction :

Le problème n'est pas de savoir ce que les symboles ou plutôt des dispositifs symboliques «signifient» mais de comprendre comment ils fonctionnent, quel ordre ils engendrent, quelles transformations ils réalisent, quel monde ils construisent. Le symbolisme relève non de la représentation mais de la performance 57.

Il est possible de rester quelque peu dubitatif devant cette interprétation plutôt abrupte, qui doit beaucoup à la lecture de Dan Sperber, d'autant plus qu'elle n'est guère attestée dans les textes eux-mêmes, mais elle a le mérite de pointer certaines ambivalences dans l'usage du concept tout au long de l'œuvre lévi-straussienne.

Pour terminer provisoirement sur cette question du sens, il reste à aborder deux points : d'une part, cerner les raisons de l'absence de l'imaginaire dans la perspective lévi-straussienne et d'autre part, comprendre ce que Descombes a nommé "l'équivoque du symbolique ", et les conséquences de cette équivoque sur LéviStrauss et Castoriadis.

(a) L'absence de l'imaginaire chez Lévi-Strauss. - À la différence du structuralisme lacanien qui a proposé le schéma RSI afin de rendre compte des relations entre réel, symbolique et imaginaire, le symbolique lévi-straussien - tel est d'ailleurs l'essentiel de la critique de Castoriadis - ne semble pas donner de place à la capacité imaginative. Il semble que, tout comme Lacan l'explicitera par la suite, l'imaginaire, réduit à une fonction purement psychologique, n'apparaît chez Lévi-Strauss que comme une puissance de leurre, une source d'aliénation comportant le risque de l'enfermement et de la chosification des figurations. L'imagination est certes une

\footnotetext{
55 Marcel Hénaff, Claude Lévi-Strauss, le passeur de sens, Paris, Perrin, 2008, p. 98.

56 Ibid., p. 111.

57 Ibid., p. 115.
} 
faculté de création infinie, mais, pour reprendre une formule d'Ortigues, «c'est ainsi que la même faculté peut donner lieu à un libre jeu de discontinuités créatrices ou à une obnubilation de la conscience dans un automatisme de répétition ${ }^{8}$ ». Ainsi que le rappelle Olivier Fressard, seul l'ordre symbolique, qui est, du fait de ses propriétés formelles, la source des impératifs, règles sociales, interdits, promesses, croyances et adhésions, peut maîtriser et intégrer la monade psychique dans le monde sensé. Pour autant, en raison de l'homologie posée par le structuralisme entre fait social et fait linguistique, il n'est d'imaginaire qu'individuel, en rupture totale avec la proposition castoriadienne d'un imaginaire social, "source sui generis et flux indépendant de représentations, d'intentions et d'affects, appartenant en propre au collectif, à l'ensemble social comme totalité59 ».

Chez Lévi-Strauss, d'une certaine façon, l'imaginaire est assimilé au désir individuel, et donc à la sphère subjective des affects et des émotions qu'il convient de mettre à distance afin de comprendre scientifiquement le fonctionnement universel de l'esprit humain classificatoire. On sait que le geste inaugural de Lévi-Strauss lorsqu'il entérine sa filiation intellectuelle avec l'héritage de l'École française de sociologie consista en une rupture avec Durkheim au nom d'une réquisition, voire annexion, biaisée de Mauss, élevé au rang de "proto-structuraliste ». Cet acte originel n'est pas sans rapport avec le refus de prendre en compte la dimension de l'imaginaire. L'affectivité, l'émotion, les sentiments qui se manifestent dans les états d'effervescence collective apparaissent comme la clé de l'analyse durkheimienne du social. Dans la lecture lévi-straussienne de ce cheminement durkheimien, celui-ci se voit interprété comme un retour pré-scientifique au "mysticisme», à une compréhension des sociétés primitives qui les caractérise par leur irrationalité, leur naturalité affective, alors qu'il faudrait au contraire les définir par leur intelligence dans l'établissement de formes de classification logique. Cette théorie durkheimienne de l'origine collective du sacré reposerait sur un faux postulat : ce ne sont pas les émotions réelles, ressenties à l'occasion des réunions ou des cérémonies qui engendrent ou perpétuent les rites collectifs, mais c'est l'activité rituelle, donc classificatoire, qui engendre l'émotion. Les sentiments, les émotions et les pulsions n'expliquent rien scientifiquement, car ils résultent selon Lévi-Strauss « toujours soit de la puissance des corps, soit de l'impuissance de l'esprit ». L'affect et l'imaginaire ne sont donc jamais des causes, lesquelles doivent être cherchées dans l'organisme (par la biologie) ou dans l'intellect (par la psychologie et l'ethnologie). L'exigence d'ordre, la classification et la logique sont donc premières par rapport tant aux expériences qu'aux émotions vécues par les hommes.

$5^{8}$ Edmond Ortigues, Le discours et le symbole, Paris, Beauchesne, 2007, p. 215.

59 Olivier Fressard, «Le symbolique et l'imaginaire », dans Cahiers Castoriadis, II. Imaginaire et création historique, dir. Philippe Caumières, Sophie Klimis et Laurent Van Eynde, Bruxelles, Facultés Universitaires Saint-Louis, 2006, p. 138-139. 
L'imaginaire et le symbolique | Stéphane VIBERT

(b) L'équivoque du symbolique. - Il est possible d'affirmer sans détour que Castoriadis et Lévi-Strauss sont deux adversaires résolus de la religion, à la fois en tant que phénomène social-historique, mais également en tant qu'objet de connaissance, à travers par exemple la catégorie du sacré. Les deux auteurs, en raison de leur ancrage matérialiste, y voient uniment un vecteur d'aliénation et d'illusion, que ce soit pour occulter le projet d'autonomie et le questionnement ouvert chez Castoriadis, ou pour voiler la quête scientifique du fonctionnement psychique chez Lévi-Strauss. Il n'est donc pas anodin que les deux auteurs, s'ils diffèrent sur le rôle de l'imaginaire, s'accordent sur la dimension essentiellement rationnelle et classificatoire des structures symboliques, fruit d'une évolution naturelle de l'espèce humaine. C'est cette reconceptualisation du terme que le philosophe Vincent Descombes, dans un article séminal intitulé «L'équivoque du symbolique 60 », a voulu souligner. Alors que Durkheim et Mauss identifiaient l'existence du social au sacré, Lévi-Strauss assimile le fonctionnement du social au langage, gommant toute différence entre le symbolisme magique et le symbolisme algébrique, conduisant ainsi à la disparition de multiples dimensions irréductibles à une combinatoire logique : le désir de puissance, l'énergie vitale, l'attachement émotionnel ou le pouvoir des affects. Ce fait avait été d'ailleurs déjà remarqué par Lacan, selon qui

Lévi-Strauss $[\ldots]$ craint que sous la forme de l'autonomie du registre symbolique, ne reparaisse, masquée, une transcendance pour laquelle, dans ses affinités, dans sa sensibilité personnelle, il n'éprouve que crainte et aversion. [...] Il ne veut pas que le symbole, même sous la forme extraordinairement épurée sous laquelle lui-même nous le présente, ne soit qu'une réapparition de Dieu sous un masque ${ }^{61}$.

De plus, cette réduction du symbolisme à l'échange réciproque, sous les auspices du paradigme communicationnel, prive le social de toute dimension verticale, dans la saisie de la normativité intrinsèque qu'il porte à l'existence, quitte à le ramener à une forme complexifiée de conventionnalisme, voire de contractualisme. Pour ce qui est de Castoriadis, l'hétéronomie, forme d'aliénation religieuse, consiste bien en une autonomisation de l'imaginaire et du symbolique, rendus hermétiques à la maîtrise humaine et à l'inventivité historique, par leur subordination à une origine inaccessible car divine. Ceci dit, son effort consistera justement, par l'élaboration et l'explicitation des sources imaginaires de l'institution sociale, à retrouver une compréhension plus fine de l'être-collectif dans sa clôture de signification et son attachement identitaire. Reste que, dans les deux cas, nous n'avons affaire qu'à un

60 Vincent Descombes, «L'équivoque du symbolique », MLN, vol. 94, $\mathrm{n}^{\circ}$ 4, French issue : perspectives in mimesis, 1979 , p. 655-675.

${ }^{61}$ La citation de Lacan est issue de ses séminaires du milieu des années 1950 : cité dans Lucien Scubla, « Le symbolique chez Lévi-Strauss et chez Lacan », Revue du MAUSS, vol. 1, n 37, 2011, p. 255. 
L'imaginaire et le symbolique | Stéphane VIBERT

monde humain et rien qu'humain, dont la limite externe n'est jamais transcendante, mais uniquement incarnée par la référence au non-sens, au néant ou au chaos.

\section{Qu'est-ce que la société : totalité autoréférentielle ou système de variations ?}

À l'évidence, Castoriadis et Lévi-Strauss ont été tous deux fortement influencés par leur lecture du courant culturaliste de l'anthropologie américaine, qui dans toute la première moitié du $\mathrm{XX}^{\mathrm{e}}$ siècle, à la suite du travail pionnier de Franz Boas, a développé une pensée originale et stimulante des rapports entre culture et personnalité individuelle, tout en cherchant à définir la configuration spécifique - le pattern - de chaque forme socioculturelle particulière, à l'aune d'une saisie comparative des différentes incarnations de l'humanité concrète. Dans sa présentation de l'individu comme « fragment ambulant ${ }^{62}$ » de sa société, et par la correspondance qu'il souligne entre l'ethos d'une société, la psyché socialisée des sujets et les significations sociales centrales, Castoriadis ne fait ainsi que réactiver l'hypothèse culturaliste d'une identité de l'être-collectif et des individus socialisés, considérés comme deux facettes d'une même réalité social-historique. Sa critique de l'anthropologie marxiste en son postulat d'un déterminisme économique infrastructurel doit beaucoup, de façon patente, à la mise en lumière ethnologique de la différence culturelle et de son irréductibilité à des motifs matériels ou naturels, ainsi que le rappellent discrètement quelques notes de bas de page renvoyant à Margaret Mead ou Ruth Benedict dans la première partie de L'institution imaginaire de la société.

On se souvient que la formation ethnologique de Lévi-Strauss s'alimenta fortement à la lecture intensive des travaux de cette même école culturaliste américaine, qui postulait un rapport étroit entre psyché individuelle et mentalité collective, d'abord à titre d'hypothèse chez Boas, puis au sein de monographies (Mead, Benedict), d'études interdisciplinaires (Sapir, Linton, Kardiner) ou de constructions théoriques (Kroeber, Herskovits). Lévi-Strauss a toujours reconnu cette dette intellectuelle, retenant notamment de Boas cette conception fondamentale, elle-même importée du romantisme allemand, selon laquelle l'existence du social réside dans l'interdépendance relationnelle de ses diverses composantes, fussent-elles a priori très hétérogènes : langue, outils, œuvres d'art, systèmes de parenté, rites, mythes. Si Lévi-Strauss reprend la notion de "culture », qui définit cette totalité organique systématisant de façon cohérente l'ensemble des faits sociaux dans un groupe donné et organisant la perception du monde pour les individus de cette société, il va toutefois la réorienter dans une perspective universaliste, rejetant théoriquement un aspect central du culturalisme, la notion de «totalité ». Lévi-Strauss reproche en effet au culturalisme d'attribuer à chaque culture une capacité de choix et une motivation esthétique analogues à celles d'un individu, dans l'éventail élargi des possibilités humaines. Le risque de l'anthropologie culturelle serait alors de faire de chaque

62 Cornelius Castoriadis, "Portée ontologique de l'histoire de la science », dans Les carrefours $d u$ labyrinthe, II. Domaines de l'homme, Paris, Seuil, 1999, p. 526. 
culture une totalité close sur elle-même, défendant son droit à l'existence dans l'ensemble des cultures possibles, en sorte que la diversité culturelle se rapprocherait de façon étrange de l'offre d'un supermarché fournisseur de valeurs ${ }^{63}$.

Certes chez Boas, la production des différences culturelles n'a jamais été conçue comme relativisme radical et irréductibilité foncière des sociétés les unes par rapport aux autres. Au contraire, Boas s'intéressait davantage à la communication entre les cultures et à la circulation de biens ou rituels réappropriés dans chaque milieu social. C'est déjà sur le modèle du langage et de la communication que Boas pensera l'explication apportée à la production des différences culturelles. Car, d'une part, le langage produit bien des différences qui singularisent les cultures, puisque c'est à partir de concepts liés à une langue que sont permis des croyances, des discours, des mythes, des chants. Mais pourtant, d'autre part, cette différence linguistique ne s'avère ni le produit d'une identité biologique ou raciale primitive ni au contraire un simple choix effectué volontairement, de manière délibérée et consciente. Car le langage ne se referme jamais sur l'interprétation consciente qu'en donne une culture particulière : le propre du langage, en effet, est qu'on peut parler sans connaître l'ensemble des règles de la langue, et en outre, toute langue est inconsciemment en rapport avec les autres langues, auxquelles elle emprunte tournures, mots, sonorités. Lévi-Strauss reconnaîtra à Boas le mérite incontestable d'avoir ainsi défini la nature inconsciente des phénomènes culturels, anticipant sur le développement ultérieur de la linguistique, et donc de l'anthropologie structurale qui s'en inspirera. Pour autant, la conception de Boas reste selon lui trop proche du romantisme, comme expression du " génie d'un peuple ", du Volksgeist, et par ailleurs, trop formaliste, au sens où le culturalisme aurait pour projet de réduire la diversité empirique à un petit nombre de formes communes, alors que le structuralisme lévi-straussien recherche les invariants dans un champ de différences, c'est-à-dire les lois qui régissent la répartition des oppositions.

De ce point de vue, le contraste avec Castoriadis est patent. Car ce dernier retient justement du culturalisme ce que Lévi-Strauss récuse, à savoir la conception de la société comme totalité socioculturelle, définie par une clôture autoréférentielle de significations imaginaires sociales cohérentes et consistantes. Se joue donc ici une différence essentielle autour de la notion de « société ». Pour Castoriadis et les culturalistes, la société comme totalité culturelle est le système particulier au travers duquel les hommes pensent le monde, et qui existe, par conséquent, en tant que collectif anonyme et impersonnel. Elle dessine une sorte de limite du pensable dont les individus n'ont pas réellement conscience, car précisément, ils ne peuvent la voir que du dedans, et n'ont nul besoin de la connaître entièrement pour y vivre pleinement. On peut à cet égard rappeler l'existence selon Castoriadis d'une

63 Frédéric Keck, Claude Lévi-Strauss, une introduction, Paris, La Découverte Pocket, 2005, p. 39. 
position première, inaugurale, irréductible du social-historique et de l'imaginaire social tel qu'il se manifeste chaque fois dans une société donnée ; position qui se présentifie et se figure dans et par l'institution, comme institution du monde et de la société ellemême 64 .

Alors que les valeurs ou les significations livrent le contenu spécifique d'un monde social-historique pour Castoriadis et les boasiens, Lévi-Strauss préfère insister sur l'action mentale en tant qu'activité spécifique qui permet la culture, laquelle définit des réseaux infinis, au sein desquels la clôture sociale n'apparaît que comme floue, relative et contingente. C'est pourquoi il défendra toujours l'intérêt des travaux neurologiques et des sciences cognitives portant sur le cerveau humain évolué - telles que la psychologie évolutionniste, la neurologie, l'intelligence artificielle - afin de mieux comprendre les faits sociaux et culturels traités par les anthropologues. Cela lui permet d'affirmer que la culture est à tous les niveaux une réinvention et une recréation, et pas simplement une transmission. Ainsi chaque personne reçoit et remet en mouvement, de sorte que la culture pour Lévi-Strauss consiste en une multitude d'actes individuels de création cognitive d'une matière plongée dans un interminable processus de création. Deux implications anti-culturalistes découlent de ce postulat « mentaliste » : d'une part, on ne peut plus parler de cultures « circonscrites » à des sociétés, qui proviendraient d'une vision de la culture comme cadre à l'intérieur duquel les actions et pensées se déploient. En ce sens, il n'existe pas de «localisation du culturel » dans une société, ainsi que le montrent dans les Mythologiques les mythes connus par des personnes se reliant les uns aux autres dans des directions complètement différentes. Seconde conséquence: la conception structuraliste renoue avec l'idée d'une anthropologie générale au sens fort, incluant le rapport avec la compréhension de l'humanité comme une espèce naturelle, dans son évolution biologique, mentale et populationnelle. Certes, il existe dans toute société des «systèmes symboliques », mais ces systèmes ne délimitent plus substantiellement aucune totalité sociale cohérente et ordonnée. Au contraire, la «culture » ainsi formée doit être reconstruite sur le plan régional de l'échange, dans un premier temps, comme ensemble de rapports structurels de symétrie et d'inversion, puis reliée à une psychologie de l'inconscient producteur de codes à un niveau supérieur, puisque ce sont les fonctions de l'esprit humain se trouvant à la base de la production des phénomènes culturels et de leur articulation logique. Ce constat a souvent été fait, à l'exemple d'un texte tardif de Pierre Clastres, qui, tout en admettant la richesse des analyses structuralistes de la parenté et de la mythologie, leur reprochait un soubassement épistémologique problématique, à savoir l'absence du social. Si les systèmes de parenté sont bien fondamentaux pour la société primitive, le corps social ne peut pourtant être rabattu essentiellement sur les liens du sang et de l'alliance. Si les mythes se pensent bien entre eux et sont susceptibles d'une lecture structurale, rien n'est encore

64 Cornelius Castoriadis, L'institution imaginaire de la société, op. cit., p. 532. 
dit sur le « lien de production et d'invention du mythe : la société65 ». Car les mythes, tout comme les rites, d'ailleurs, qui en constituent l'expérience privilégiée sans pouvoir être soumis à une codification structurale, « constituent le discours de la société primitive sur elle-même 66 ", à travers une dimension sociopolitique qui échappe à l'abstraction formaliste. D'où ce jugement sans appel de Clastres : «ce discours élégant, souvent très riche, ne parle pas de la société. Il en est du structuralisme comme d'une théologie sans dieu : c'est une sociologie sans société67 ».

Ceci étant dit, il existe dans l'œuvre lévi-straussienne une position philo-politique principielle qui, sans entrer directement en contradiction avec l'épistémologie structuraliste, semble néanmoins laisser apparaître des tensions fortes avec cette « absence » de la société : c'est tout le discours sur la critique du progressisme occidental, de l'homogénéisation culturelle et de l'universalisme abstrait. Ainsi que l'a bien noté Tzvetan Todorov68, la critique théorique du culturalisme, adossée à une pensée du fonctionnement universel de l'esprit et à une affirmation résolue de l'unité physico-psychique de l'humain, semble souvent refluer au profit d'énoncés normatifs d'orientation relativiste, où la notion même de "société " ressurgit comme lieu de différence et de spécificité culturelle irréductible, sans pourtant être véritablement conceptualisée. Ainsi ce jugement sans ambages dans Tristes tropiques :

Aucune société n'est foncièrement bonne ; mais aucune n'est absolument mauvaise; toutes offrent certains avantages à leurs membres, compte tenu d'une iniquité dont l'importance paraît approximativement constante 69 .

La critique lévi-straussienne de la logique cartésienne de "l'homme maître et possesseur de la nature » le conduit à une critique de l'humanisme, dessiné sous les traits d'une domination occidentale à la fois ethnocentriste sur le plan des valeurs et matérialiste en vertu de sa supériorité technique ${ }^{70}$. Dans sa double dénonciation du christianisme et du cartésianisme comme sources de cette extraversion civilisationnelle, Lévi-Strauss ne se retrouve pas très loin du Castoriadis contempteur de l'imaginaire de maîtrise rationnelle illimitée, qu'il identifie parfois aux mêmes origines, pour mieux les opposer au projet d'autonomie germé en Grèce antique. Ce

65 Pierre Clastres, Recherches d'anthropologie politique, Paris, Seuil, 1980, p. 159.

66 Ibid., p. 160.

67 Idem.

68 Tzvetan Todorov, « Lévi-Strauss entre universalisme et relativisme », Le Débat, vol. 5, n 42, 1986, p. 173-192.

69 Claude Lévi-Strauss, Tristes tropiques, Paris, Plon, p. 463.

$7^{0} \mathrm{Ce}$ qui aboutit chez l'anthropologue à des raccourcis que Castoriadis récuserait au nom de la « double nature » de la modernité : " Toutes les tragédies que nous avons vécues, d'abord avec le colonialisme, puis avec le fascisme, enfin les camps d'extermination, cela s'inscrit non en opposition ou en contradiction avec le prétendu humanisme sous la forme où nous le pratiquons depuis plusieurs siècles, mais, dirais-je, presque dans son prolongement naturel». Claude Lévi-Strauss, cité dans Tzvetan Todorov, «Lévi-Strauss entre universalisme et relativisme », op. cit., p. 177. 
dédoublement axiologique castoriadien (projet d'autonomie $v s$ rationalité instrumentale) apparaît autre chez Lévi-Strauss, qui réintroduit une perspective autocritique à l'intérieur du cheminement occidental par l'apparition de l'ethnologie et la découverte de l'autre, comme réponses à la mauvaise conscience née de la domination coloniale. Reste que là où Castoriadis fait de l'humain le cœur de la création radicale et de l'imaginaire instituant, l'orientation anti-humaniste de Lévi-Strauss débouche plutôt sur un rabaissement de l'humain au niveau de la vie, et vers une sorte de naturalisme à tonalité bouddhiste qui n'est pas sans résonance avec le discours écologiste actuel de la décroissance et du localisme.

Un peu étrangement, donc, Lévi-Strauss retrouve au niveau de son discours normatif divers éléments d'un relativisme culturel rejeté comme source théorique. C'est pourquoi, au final, Castoriadis et lui semblent relativement proches dans l'examen des différences du rapport à l'historicité entre sociétés hétéronomes et autonomes, ou sociétés froides et chaudes pour reprendre le vocabulaire lévi-straussien. De même en ce qui concerne certaines déclarations provocantes de Lévi-Strauss dans « Race et culture $^{71} \gg$ afin de soutenir le maintien de la diversité culturelle, quitte à passer par un certain degré d'indifférence, voire de xénophobie, assertions qui là encore font écho à diverses remarques de Castoriadis dans ses " réflexions sur le racisme ", qui voit une certaine pente naturelle à l'humanité, selon laquelle "l'infériorité des autres n'est que l'autre face de l'affirmation de la vérité propre des institutions de la société-Ego ${ }^{72}$ », même si en dernière instance, une société aux prétentions autonomes ne peut que vouloir convertir les autres, à l'aune de l'universalisme sous-tendu par cette visée. Dans ces analyses, tant Castoriadis que Lévi-Strauss tentent donc difficilement d'articuler une version méthodologique du relativisme culturel comme évidence de la pluralité des totalités socioculturelles avec une perspective universaliste non hégémonique, soit sous les traits d'une exemplarité du projet d'autonomie, soit d'un mouvement civilisateur ethnologiquement assez conscient de l'importance de sa diversité interne. Dans les deux cas, c'est bien un horizon universaliste qui vient surplomber la différence culturelle, à partir d'un logon didonai, un « rendre compte et raison » orienté par le discours de la science73 sans s'y dissoudre entièrement.

Ces hésitations lévi-straussiennes autour de la notion de "société » comme univers cohérent et consistant de sens deviennent d'autant plus perceptibles lorsqu'on examine la transformation du structuralisme en post-structuralisme, lequel ne fait justement qu'extrapoler la critique épistémologique à l'encontre de toute totalité

${ }^{71}$ Claude Lévi-Strauss, « Race et culture », Revue internationale des sciences sociales, vol. $23, \mathrm{n}^{\circ} 4$, 1971, p. 647-666.

${ }^{72}$ Cornelius Castoriadis, «Réflexions sur le racisme », dans Les carrefours du labyrinthe, III. Le monde morcelé, Paris, Seuil, 1990, p. 37.

73 Lévi-Strauss défend « le savoir scientifique qui, quels que soient les maux qu'ont entraînés ses applications et ceux plus accablants encore qui s'annoncent, n'en constitue pas moins un mode de connaissance dont on ne saurait contester l'absolue supériorité » : Claude Lévi-Strauss, cité dans Tzvetan Todorov, «Lévi-Strauss entre universalisme et relativisme », op. cit., p. 190. 
synthétique, sous les traits d'une dénonciation de l'identité comme clôture de référence. Jacques Derrida 74 , par exemple, insistera sur les deux manières concurrentes et distinctes de lier les différences entre elles dans un système relationnel : comme rapports entre écarts différentiels dans le structuralisme, qui reste alors quantitatif et déterministe $v s$ comme mouvement interne de la trace qui porte le texte à ses limites et impulse sa dynamique interne jusqu'à le faire potentiellement exploser (« différance »). En raison de ses « résidus» modernes et humanistes, telle une «métaphysique de la présence » à fondement logocentrique, le structuralisme resterait donc pour Derrida hanté par la nostalgie d'une unité originaire, d'où le pessimisme de Lévi-Strauss quant à l'avenir des cultures et civilisations rattrapées par l'homogénéité75. Pour Frédéric Keck, commentateur de Lévi-Strauss, la critique de Derrida montre toute l'ambiguïté du "post-structuralisme », qui désigne tantôt un dépassement du structuralisme par la déconstruction, tantôt une reprise de ses intuitions fondatrices. Derrida est en fait celui qui aura poussé à l'extrême la logique interne du structuralisme, vers une remise en cause encore plus radicale de tout substantialisme, de toute essentialisation, de tout fondement, dans le sens d'une évacuation de tout signifié, grâce aux termes du « jeu» et du «supplément». Toute l'attention de Derrida se porte sur les dysfonctionnements plutôt que les régularités, définissant toute structure comme un « jeu infini de différences », traquant toutes les transcendances cachées dans les divers couples binaires (nature/culture, voix/écriture, sensible/intelligible) censés organiser le rapport au monde.

Pour finir sur ce point du rapport au concept de "société », on peut évoquer les analyses de deux anthropologues contemporains parmi les plus importants, associés à ce qui est désormais nommé le « tournant ontologique » de la discipline ${ }^{76}$, le français Philippe Descola et le brésilien Eduardo Viveiros de Castro, qui décèlent chez LéviStrauss deux structuralismes, dont les filiations finissent par s'opposer aujourd'hui. Ces deux structuralismes se distinguent par le changement opéré au sein des écrits lévi-straussiens concernant la définition de la notion de «transformation », essentielle à la méthode structurale77. Selon Descola et Viveiros de Castro, les deux définitions de la structure ont toujours cohabité dans les écrits de Lévi-Strauss, mais avec une accentuation différente. Si l'on suit Viveiros de Castro78, on trouve d'une part une définition de la transformation structurelle comme opération logique et algébrique, notamment

\footnotetext{
74 Voir, sur ce rapport Lévi-Strauss/Derrida, les interprétations particulièrement éclairantes de Frédéric Keck, dans Claude Lévi-Strauss, une introduction, op. cit.

$75 C f$. Jacques Derrida, «La structure, le signe et le jeu dans le discours des sciences humaines ", dans L'écriture et la différence, Paris, Seuil, 1967.

76 John. D. Kelly, «The ontological turn in French philosophical anthropology », Hau : Journal of ethnographic theory, vol. 4, no 1, 2014, p. 259-269.

$77 C f$. Philippe Descola, "L'arbre et la grille. Remarques sur la notion de transformation dans l'anthropologie structurale ", dans Claude Lévi-Strauss et ses contemporains, dir. Pierre Guenancia et Jean-Pierre Sylvestre, Paris, Presses Universitaires de France, 2012, p. 181-194.

78 Cf. Eduardo Viveiros de Castro, «Devenirs du structuralisme », dans Métaphysiques cannibales, Paris, Presses Universitaires de France, 2009.
} 
dans les Structures élémentaires de la parenté : c'est un modèle donc «algébricocombinatoire », procédant par permutations syntaxiques, qui en quelque sorte considère la structure comme un ensemble potentiellement clos et à l'ordonnancement rationalisable. D'autre part, de manière évidente surtout dans les Mythologiques, la transformation structurelle se rapprocherait d'une opération esthétique par déformation et déphasage, selon un modèle cette fois "topologique et dynamique », qui procède par innovation sémantique ou condensation morphogénétique. Selon Viveiros de Castro, Lévi-Strauss dans son œuvre va de plus en plus appuyer le caractère discontinu de la structure, qui finit par se rapprocher ainsi du rhizome deleuzien, cette arborescence infinie, une gigantesque toile sans centre, ni origines, ni frontières, un réseau traversé par de multiples flux sémiotiques, matériels et sociaux :

peut-être devrait-on dire qu'il y a deux usages différents du concept de structure dans cette œuvre : en tant que principe transcendantal d'unification, loi formelle de l'invariance; et en tant qu'opérateur de divergence, modulateur de variation continue (variation de variation). La structure comme combinatoire grammaticale fermée ou comme multiplicité différentielle ouverte79.

Viveiros de Castro fait ainsi quasiment de Lévi-Strauss un post-structuraliste avant la lettre 80 : c'est la disparition du concept même de "société » qui se joue à travers cette évolution, et le passage d'une ontologie moderne à une perspective postmoderne. S'il serait certainement tout à fait rétif à la terminologie d'un " principe transcendantal d'unification ", nul doute néanmoins que toute l'œuvre de Castoriadis témoigne en faveur d'une interprétation du social-historique faisant droit aux significations imaginaires sociales centrales qui spécifient et particularisent tout univers culturel original, caractérisé par une clôture relative qui en oriente la conception du monde et le constitue comme forme singulière, comme eidos irréductible, quelles que soient par ailleurs les relations d'interdépendance qui l'attachent aux contraintes naturelles et aux échanges inter-sociétaux.

\section{Conclusion}

À l'évidence, bien d'autres points auraient pu être soulevés pour faire discuter deux œuvres ambitieuses, complexes, englobantes et multidimensionnelles. Concluons donc provisoirement par deux contrastes fondamentaux et une question ouverte.

\footnotetext{
79 Ibid., p. 181.

80 «Les relations qui constituent les récits amérindiens, plutôt que de former des totalités combinatoires en distribution discrète, en variation concomitante et en tension représentationnelle avec les realia socioethnographiques, exhibent de façon exemplaire les principes de "connexion et hétérogénéité", "multiplicité", "rupture assignifiante" et "cartographie" que Deleuze et Guattari contraposeront aux modèles structuraux au nom du concept de "rhizome" - ce concept qui était supposé être le nom propre de l'antistructure, le cri de guerre du post-structuralisme ». Ibid., p. 177.
} 
Premier contraste : là où Lévi-Strauss fonde sa théorie sur une ontologie moniste à soubassement naturaliste et à tendance entropique, Castoriadis entreprend de creuser la distinction phusis/nomos afin de proposer une ontologie de la création, qui maintient fortement la spécificité de l'humanité comme lieu d'un imaginaire instituant vecteur de l'Être comme temps, et donc comme processus à la fois différenciateur et contingent. Second contraste : là où Lévi-Strauss conçoit l'imaginaire comme désir subjectif et le symbolique comme codage inconscient, à la fois culturel et universel, Castoriadis subordonne le symbolique, comme dimension ensidique, rationnelle et étayée sur la première strate naturelle, à l'imaginaire comme puissance de signification, englobant et orientant les nécessités de fait par des relations arbitraires et immotivées, sinon selon le social-historique lui-même.

À partir de ces deux contrastes, il est possible de soulever une interrogation là encore décisive pour cerner les approches en présence, notamment au plan d'une conception générale qui noue ensemble hominisation et humanisation : c'est évidemment le rapport du monde humain social à la nature, plus précisément à son ancrage biologique et son inscription dans un écosystème lui-même évolutif. Car il faudrait s'interroger sur les figures que prend chez Castoriadis et Lévi-Strauss l'envers du sens, autrement dit le Chaos, l'Abîme et le Sans-Fond pour l'un, le nonsens pour l'autre. Dans les deux cas, le sens surgit à partir de son opposé, soit comme continuité structurée, soit comme discontinuité imaginaire. Mais chez les deux auteurs, les conditions matérielles ou biologiques ne se présentent jamais comme une matière brute, un chaos informe sur lequel l'esprit humain agirait comme mise en ordre totalement arbitraire, car la nature se caractérise par une structure propre et intelligible, au moins partiellement déjà ordonnée, au sein de laquelle chaque culture ou monde social-historique prélève certains éléments de préférence à d'autres, se conférant une signification propre et un style particulier d'être-au-monde. Il n'y a donc pas de niveau purement empirique auquel se surajouterait la signification, qui est toujours déjà donnée d'emblée dans l'existence sociale. Dès qu'il y a société humaine, les conditions matérielles, qui certes obéissent à des mécanismes propres, sont reprises et transformées en un système qui valorise et donne des significations autres que biologiques. Resterait à savoir si Castoriadis accepterait, à partir de ses recherches sur l'apeiron et l'indéterminé, une formulation telle que celle-ci : " dans ma perspective, le sens n'est jamais un phénomène premier : le sens est toujours réductible. Autrement dit, derrière tout sens il y a un non-sens, et le contraire n'est pas vrai. Pour moi, la signification est toujours phénoménale 81 ».

81 Claude Lévi-Strauss, « Réponses à quelques questions », op. cit., p. 637. 